\title{
Virtual Reality for Teaching and Learning in Crime Scene Investigation
}

\author{
Richard Mayne $^{a, b, 1} \&$ Helen Green ${ }^{a}$ \\ ${ }^{a}$ Department of Applied Sciences, Faculty of Health and Applied Sciences, University of the West \\ of England, Bristol, BS16 1QY, United Kingdom \\ ${ }^{b}$ Mayne Bioanalytics, Bristol, BS34 5BH, United Kingdom
}

\begin{abstract}
Simulated crime scene investigation is an essential component of forensic science education, but its implementation poses challenges relating to cost, accessibility and breadth of experience. Virtual reality (VR) is an emerging technology which offers exciting prospects for teaching and learning, especially for imparting practical skills. We document here a multidisciplinary experimental study in which a bespoke VR crime scene app was designed and implemented, after which it was tested by both undergraduate student and staff/postgraduate student cohorts. Through both qualitative and quantitative analyses, we demonstrate that VR applications support learning of practical crime scene processing skills. VR-based practical sessions have the potential to add value to forensic science courses, through offering cost-effective practical experience, the ability to work in isolation and in a variety of different scenarios. Both user groups reported high levels of satisfaction with the process and reports of adverse effects (motion sickness) were minimal. With reference to user feedback, we proceed to evaluate the scalability and development challenges associated with large-scale implementation of VR as an adjunct to forensic science education.
\end{abstract}

Keywords: Higher Education, Pedagogy, Forensic Science, VR, Learning Technologies, Crime Scene

\section{Introduction}

The teaching of crime scene investigation (CSI) skills is an indispensable component of undergraduate and postgraduate Forensic Science courses. Indeed, the UK Chartered Society of Forensic Sciences mandates that students undertaking accredited courses achieve a certain standard of competency in their practical skills through simulated investigation [1]. Whilst higher education practitioners usually provide extensive training in mock-up crime scenes, there are several key limitations to on-site

\footnotetext{
${ }^{1}$ Corresponding author: Richard.Mayne@uwe.ac.uk
} 
practical sessions, including high costs of operation (including academic and technician time), lack of accessibility for differently-able students and limitations on the number and type of scenarios that can be offered by a single facility. This investigation evaluates the use of a novel technology, virtual reality (VR), under the hypothesis that digital methods may be used as an adjunct to teaching and learning of practical crime scene skills in forensic science.

VR technologies encompass a range of digital tools that are used to immerse the user in a digitally-generated world. Typically, this involves the user wearing a headset that contains stereoscopic projectors which are capable of producing a threedimensional (3D) image; more advanced hardware usually have linked positionallytracked controllers and various systems for monitoring user movement, such as frontmounted cameras or external infrared laser projectors.

Although VR is not novel, it has only been in the last decade that hardware advances have allowed for the generation of 3D worlds whose graphical resolution and frame rates can approach those of 'real life' and hence offer an immersive and worthwhile experience. Such technologies are still considered as inaccessible to many home users on the basis of cost, with headsets requiring either a powerful computer to run their applications or otherwise miniaturised on-board batteries, processing and graphical hardware and storage media. The recent release of standalone headsets that cost approximately GBP 500 per unit, however, suggests that VR technologies are now affordable options for use in higher education scenarios.

We approach this investigation taking it as read that digital simulations are an effective and widely-used tool in higher education, especially for skills-based learning. Whilst research into the pedagogical applications of VR are expanding rapidly, with reviews and speculative studies gradually emerging in the life sciences domain $[2,3]$, experimental studies into applied teaching and learning using fully-interactive VR applications are relatively sparse, although notable exceptions do exist, see Refs. $[4,5,6]$. The reasons for this apparent exiguity likely stems from the financial and skills investments that VR development requires, but as open access development tools continue to gain traction, this investment is reducing steadily. This is emblematic of the importance of multidisciplinary research uniting the applied sciences with digital technologies.

At the time of writing, few studies on the application of VR to forensic science education exist, although limited research demonstrates that VR is an effective tool in the education of criminal law [7]. In 2019, Leung and Blauw [8] reported on experiences of students using augmented reality (a related technology) in the education of digital forensics. In the same year, Karabiyik et al. [9] developed a conceptual framework for training incident first responders and digital forensic investigators (which was not utilised in the current study). To our knowledge, ours is the first experimental study into the use of VR for CSI education.

This article is structured as follows. First, we present an experimental study into the use of VR technologies for enabling teaching and learning in CSI scenarios, after 
which we present and discuss our results with regards to CSI education. Finally, we conclude by discussing the costs, limitations and challenges for developing VR applications for higher education.

\section{Methods}

\subsection{Participant selection}

Two participant groups were selected. Firstly, a group of first year undergraduate students (UGS) studying for a forensic science BSc Honours or MSci degree $(n=$ 10). At the point of being tested, this cohort had received a single 'traditional' session in a mock-up crime scene investigation, so were assumed to have a basic understanding of crime scene investigation but had no experience of doing so in an unsupervised environment. The second group consisted of staff and postgraduate researchers $(\mathrm{S} / \mathrm{PGR})$ who were all involved in teaching or researching in forensic science or related fields $(n=8)$. This group were assumed to be well-informed and confident in crime scene investigation scenarios, in either pedagogical or professional contexts. No reimbursements or incentives were offered for test participation.

\subsection{Hardware}

VR testing was conducted on Oculus Quest 128 Gb headsets (Facebook, USA). These devices operate independently of a computer, using internal batteries and graphics processing units; coupled with their six degree of freedom movement tracking via internal gyroscopes, front-mounted cameras and positionally-tracked controllers (Fig. 1), this allowed users to navigate freely around their environment with minimal restrictions or disruptions to immersion. Devices were operated offline, in developer mode, following being built on a high-performance development machine (see Supplementary Information (SI) Section 1.1 for specification).

\subsection{Software \& App Design}

A virtual crime scene application (app) was created in Unity version 2019.2.15f1 (Unity Technologies, Denmark) via its Oculus and Android Studio (Google, USA) software development kits. The virtual environment was designed according to schematics for a room in the authors' institution's dedicated CSI 'house', using a combination of open source 3D assets and custom-designed models, the latter of which were created in Blender version 2.81 (Blender Foudnation, Netherlands).

The room, which was modelled as a lounge (Fig. 2), contained a bookcase, sofa, two chairs, a coffee table, a lamp and a flat-screen television on a stand; the room was further lit via a ceiling-mounted light and through two windows, the exterior skybox for which was set to a level of illumination consistent with a bright United Kingdom day. Lightmaps were baked prior to the application build to maintain performance, which resulted in an average framerate of 59 in stress tests.

Three pieces of 'evidence' were planted around the lounge: glass shards under the window, a small bloodstain on the carpet between the two chairs and a knife, partially 


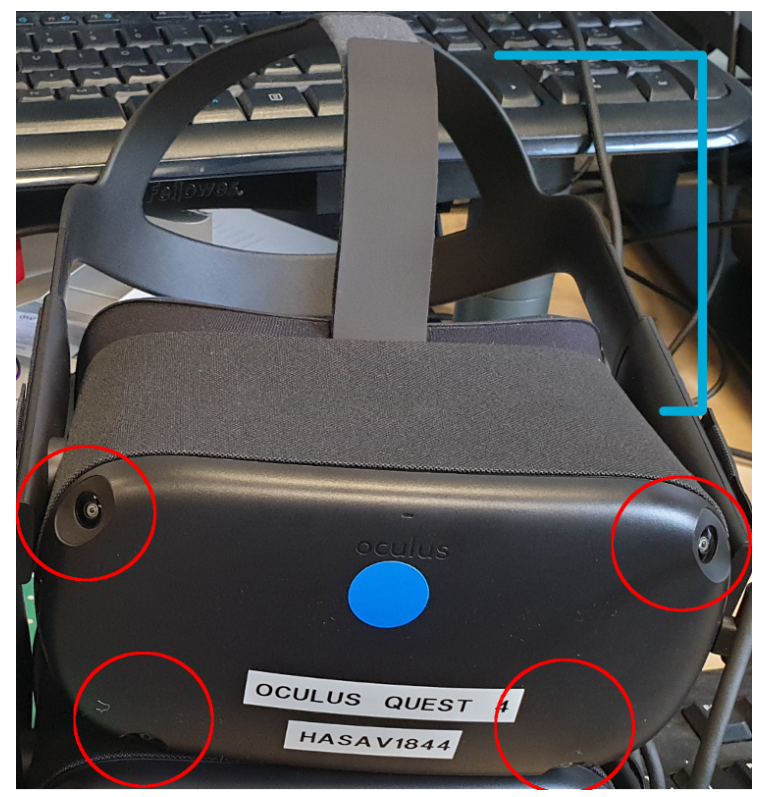

(a)

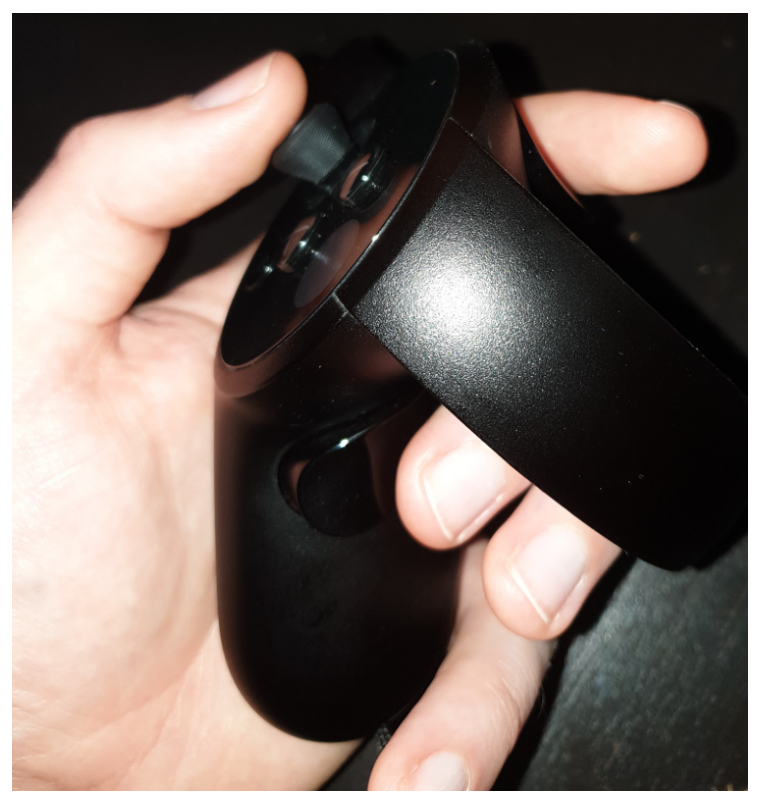

(b)

Figure 1: Photographs of Oculus Quest hardware. (a) Headset. Red circles indicate front-mount cameras for positional tracking, blue shows headband region. (b) LHS touch controller. Note the thumbstick, index finger trigger and middle finger trigger.

hidden on the bookcase (Fig. 3). Several evidence markers were also present on the coffee table, which the users could pick up and place on the evidence sources via the middle finger squeeze-grip buttons (see Fig. 1b). A photography tool was added using the headsets' on-board screenshot capability, which was used for processing the crime scene (see Section 2.4).

Users were able to navigate through their environment in two ways: firstly, they could opt to walk around their environment, which was detected and translated into VR movements by their headset's various sensors. Secondly, they could use their left hand controller's thumbstick to move around, in a manner similar to a games console controller; the right hand controller stick could be used to 'snap turn' $45^{\circ}$ in either direction, which is a recommended 'quality of life' option for reducing user motion sickness. It was possible to use both of these control schemes in combination.

Design considerations for differently-able users were as follows: a high-contrast mode was available for users with partial or complete colourblindness; the dual control schemes permitted users to complete the test standing or sitting; headset cushion adapters were available for glasses wearers.

A video walkthrough of the virtual crime scene is included in SI Section 1.2.

\subsection{Test Design}

Three categories of measurement were collected: attainment of learning outcomes (LOs), questionnaire scores and user feedback. 


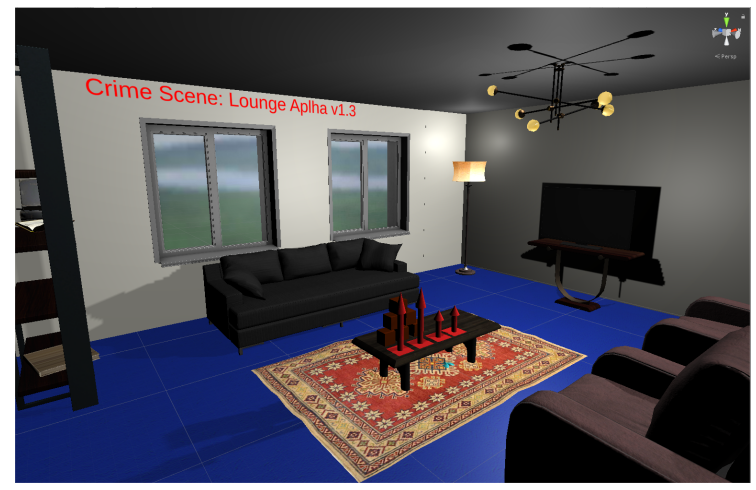

(a)

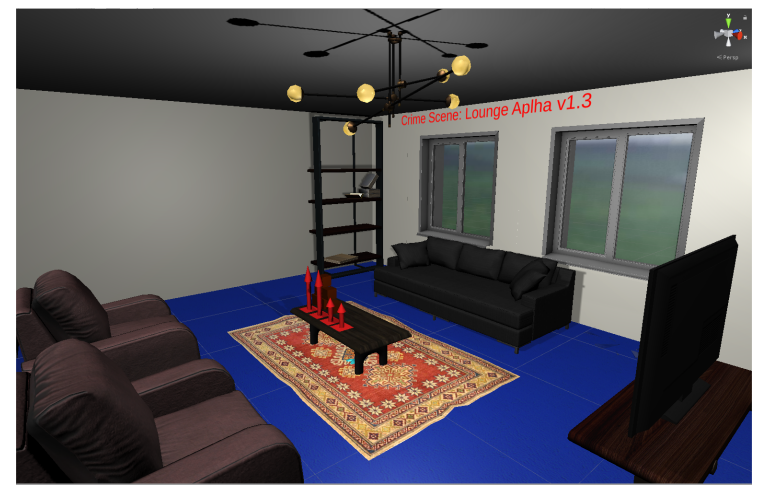

(c)

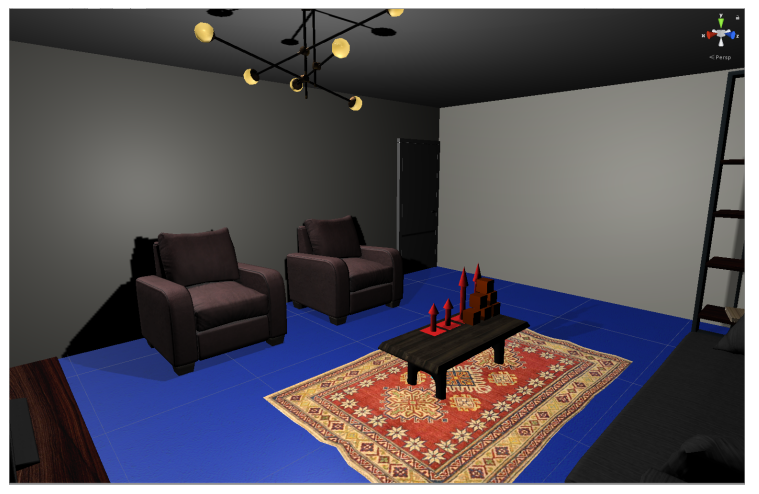

(b)

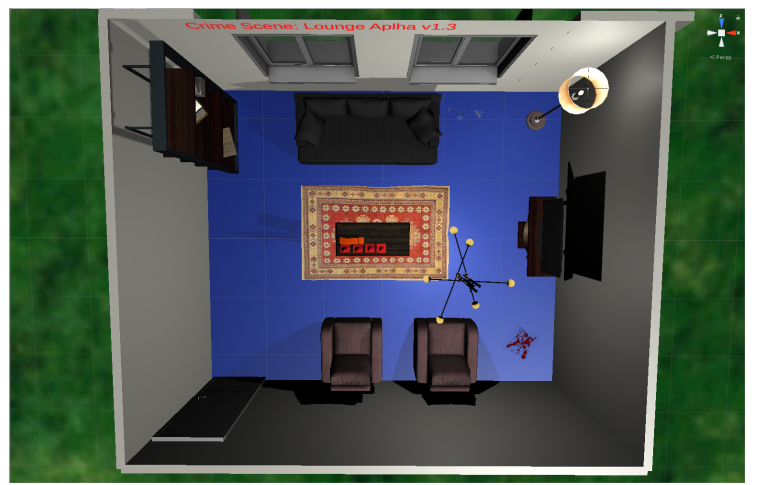

(d)

Figure 2: Renderings of virtual reality environment. (a-c) Room corners; note the various 'markers' on the central table that users can grab and place. (d) Birds-eye view. 


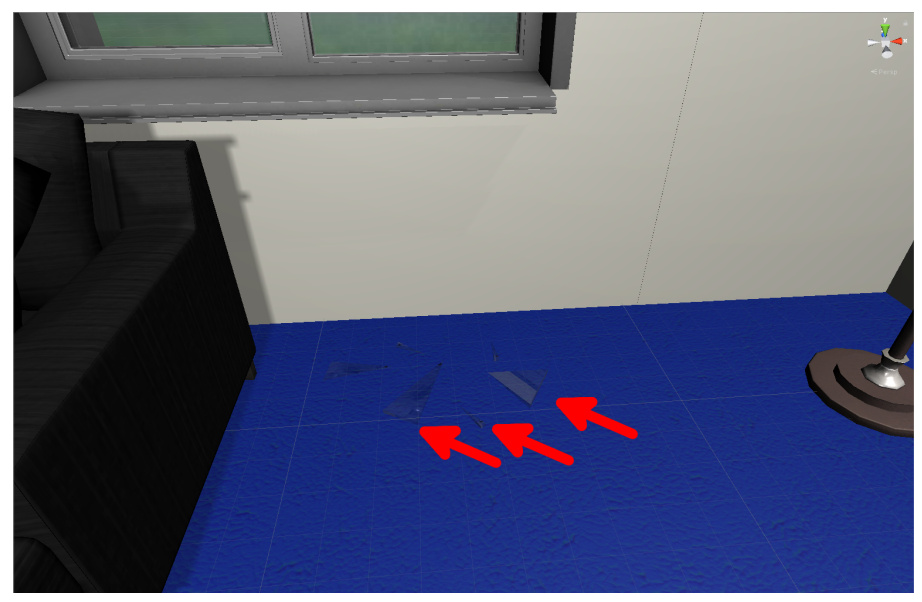

(a)

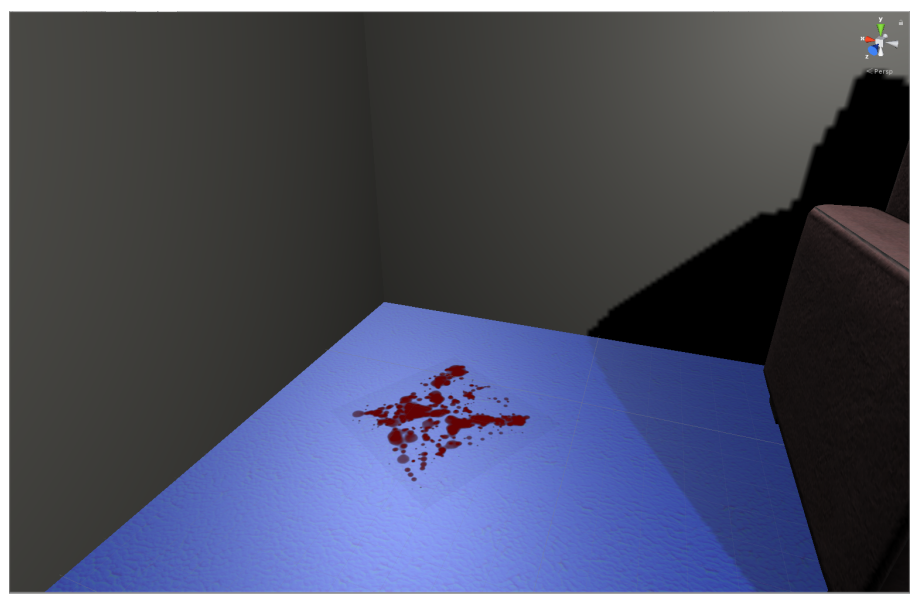

(b)

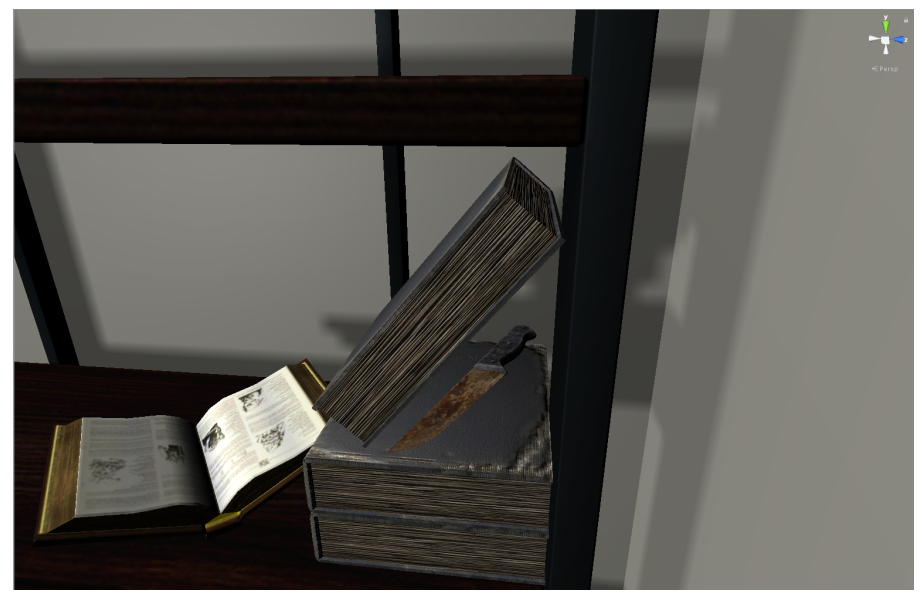

(c)

Figure 3: Renderings of three pieces of 'evidence' in virtual reality application. (a) Glass shards under a window (arrowed). (b) Blood stain. (c) Knife on bookshelf. 
Before experiments were conducted, users were given a safety briefing and introduction to VR headset fitting and controls before finally, a scenario was introduced (see SI Section 1.3 for full scenario).

The users were then instructed to process the crime scene, making use of the photo tool and evidence markers. Time in the VR environment was limited to 20 minutes, after which users were asked to make notes on the following four criteria, which were directly linked to the LOs for the task:

1. What they did to process the crime scene; LO attainment was linked to taking photographs of the scene from multiple angles, placing the evidence markers and taking further photographs of the evidence in situ.

2. What evidence they found; LO was only achieved if users found all three pieces.

3. What they hypothesised had occurred; LO was attained if the users noted a sensible hypothesis relating the evidence found to the scenario briefing.

4. What a logical next step for analysis would be; LO was attained for any suitable answer, such as checking for finger marks.

These LOs were all taken from the authors' institution's syllabus for first year Forensic Science students, with attainment scores of 0-4 roughly corresponding to fail, third, two:two, two:one and first classifications for the skills component of their course.

Following, users were asked to complete a questionnaire with eight questions, each on a conventional Likert scale of 1-5 (see SI Section 1.4). Finally, users were asked to note any comments they felt were pertinent, with specific regards to suggested improvements or adverse experiences.

Testing was conducted in large, otherwise-empty studios, whose free space allowed for a maximum of four concurrent users. Each user was allocated a $2 \times 2 \mathrm{~m}$ area in which to complete the experiment, which was delineated by both the headset's 'guardian' system - which warns users when they approach the limits of their predefined safe-zone - as well as markings on the floor, which the users were made aware of before putting the headset on. Each user's area was separated from other users' areas by a $1 \mathrm{~m}$ exclusion zone. Users were asked to not communicate with each other whilst the tests were in progress.

\subsection{Analysis}

Experiment outcome was gauged through user attainment of LOs, questionnaire responses and comments. LO attainment was assessed by comparing median scores between cohorts. Questionnaire responses were analysed both intra- and inter-group, through using single-sample, two-tail Wilcoxon tests and two-tail Mann-Whitney tests, respectively. Analyses were performed in Python, using the SciPy library. Comments were manually tabulated and discussed. 


\section{Results}

Median LO attainment is shown in Tab. 1; there was a significant difference in the number of LOs achieved between UGS (3/4) and S/PGR (4/4) groups.

The questionnaires demonstrated (Tab. 2) that both students and staff found: the pre-test briefing ample (Q1); the controls intuitive (Q4); the app immersive (Q5); the app had suitable resources to enable learning about CSI (Q6); using the app was enjoyable (Q7). Staff found it easy to navigate around the crime scene, whereas students had no strong opinion (Q2). Students found the app did not induce motion sickness, while staff reported mixed results (Q3). Neither students nor staff had strong opinions on which control scheme was best (Q8).

Comparison of median scores between UGS and S/PGR groups demonstrated a significant difference in responses for only one question, Q5, indicating that staff found the app more immersive than students did (Tab. 2).

\begin{tabular}{l|ll|l}
\hline & UGS & S/PGR & $p$ \\
\hline Med & 3 & 4 & 0.003 \\
\hline
\end{tabular}

Table 1: Median learning outcome attainment of a total of 4, for first year undergraduate students (UGS) and staff and postgraduate researcher (S/PGR) groups. Also shown is the $p$ value for a Mann-Whitney test showing a significant difference between these medians $(p<0.01)$.

\begin{tabular}{lc|llllllll}
\hline Group & Test & Q1 & Q2 & Q3 & Q4 & Q5 & Q6 & Q7 & Q8 \\
\hline UGS & Med & 4.5 & 4.0 & 2.0 & 4.0 & 4.0 & 4.5 & 5.0 & 3.5 \\
& $p$ & $0.006^{\dagger}$ & 0.058 & $0.030^{*}$ & $0.014^{*}$ & $0.022^{*}$ & $0.014^{*}$ & $0.006^{\dagger}$ & 0.116 \\
S/PGR & Med & 5.0 & 4.5 & 2.6 & 4.3 & 5.0 & 4.3 & 4.5 & 3.0 \\
& $p$ & $0.014^{*}$ & $0.014^{*}$ & 0.529 & $0.022^{*}$ & $0.014^{*}$ & $0.036^{*}$ & $0.014^{*}$ & 0.423 \\
\hline \hline Diff. & $p$ & 0.156 & 0.361 & 0.195 & 0.697 & $0.017^{*}$ & 0.730 & 0.070 & 0.566 \\
\hline
\end{tabular}

Table 2: Questionnaire Likert score analyses. (Upper) Median (Med) questionnaire scores and single-sample Wilcoxon test $p$ values, for first year undergraduate students (UGS) and staff and postgraduate researcher (S/PGR) groups. (Lower) $p$ values for Mann-Whitney tests comparing the differences (Diff.) between questionnaire medians of both cohorts. $* p<0.05, \dagger p<0.01$

One $(10 \%)$ of the UGS group reported mild motion sickness, compared with four (50\%) of the S/PGR group who experienced motion sickness, one case of which was severe. The most frequent comments received were reports of satisfaction with the process, followed by suggested new features, followed by reports of motion sickness (full list in S.I. Section 1.5). Suggested extra features included the option for blood pattern analysis, being able to move furniture, including rulers for marking evidence and general fixing of minor bugs. The UGS group reported more bugs, conversely the $\mathrm{S} / \mathrm{PGR}$ group reported more suggested features. 


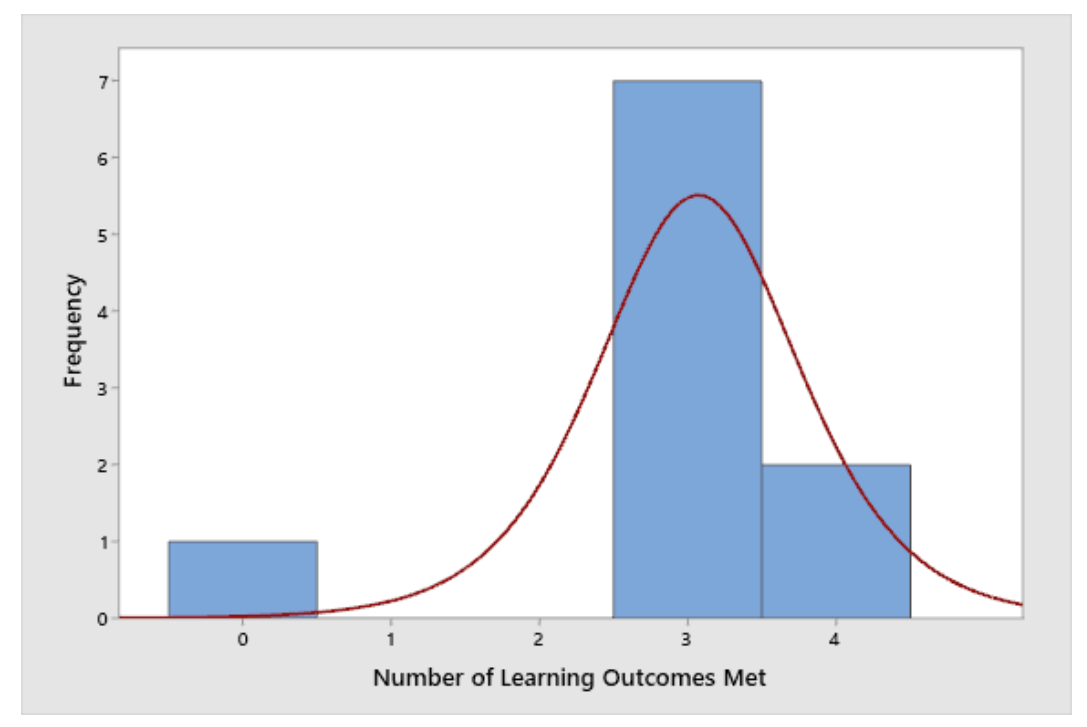

Figure 4: Histogram to show distribution of number of learning outcomes met in undergraduate student (UGS) group, with overlaid trend line.

\section{Discussion}

\subsection{Suitability for Teaching \& Learning}

Analysis of LO attainment demonstrated that the VR app was fit for purpose, with all but one of the UGS group attaining a score of 3-4 (Fig. 4). Anecdotally, this distribution had a higher median than would normally be expected for this variety of assessment when applied to first year students, although the sample was likely to have been composed disproportionately of higher-achieving students. Every user in the S/PGR group achieved all LOs.

The most frequent comments made were reports of having enjoyed the experience; as the users were not prompted to do so and the questionnaire data further indicated a high degree of satisfaction (Q5-7), these were taken as indications that VR is a suitable tool for enhancing teaching and learning in this context. We would expect student satisfaction to be a significant factor in the effectiveness of any teaching and learning activity $[10,11,12]$.

Questionnaire responses for other questions indicated that the control scheme was suitable for purpose (Q2, 4, 8), although reports from the UGS group that the VR environment was neither easy nor difficult to navigate are somewhat difficult to interpret. With reference to user comments, however, it is possible that the UGS users would have liked to have had the option to explore the virtual environment without use of the controllers, which was unfortunately impossible due to the room scale, $3 \times 5 \mathrm{~m}$, being larger than the available studio space. 


\subsection{Safety and Tolerability}

The most commonly-reported problems with the use of VR technologies are safety issues arising from movement whilst sight-deprived and motion sickness [13]. No safety issues occurred during testing and only one user reported severe motion sickness.

It was reasoned that the cause for the S/PGR group reporting a higher degree of immersion than students could be a reflection of novel technologies being less novel to the UGS cohort; all UGS users reported during the controls briefing, for example, that they had used a games console controller previously, compared with only half of the S/PGR group reporting this (data not shown). Whilst it's tempting to theorise on how prior exposure to computer games impacts on user experience, such as its apparent correlated with lower rates of motion sickness in the UGS group, no other significant differences in questionnaire scores were observed between the two cohorts.

It is worth noting that $25 \%$ of S/PGR users did the test whilst sat down all of whom reported at least mild motion sickness - compared with $0 \%$ in the UGS group. This indicates that designing quality-of-life improvements for sedentary position users are a sensible decision for future development: possible modifications include 'teleport'-type motion, reducing avatar acceleration and removing the user's peripheral vision whilst their avatar is in motion.

\subsection{Cost-benefit Analysis}

We estimate the setup cost for a robust virtual reality suite to be GBP 8,000, with consequent cost of practicals to be circa GBP 900 per scene for a cohort size of 90 (see SI Section 1.6). Conversely, we calculated the cost of a typical practical session in a simulated crime scene to be GBP 1800 for the same cohort.

We therefore find that, after an admittedly substantial initial outlay, the use of $\mathrm{VR}$ is a cost-effective solution for teaching practical CSI skills. Whilst it is beyond the remit of this investigation to speculate whether practical sessions could be partially replaced with VR sessions, it is feasible to assume that, if a single institution provides four sessions per year to four levels of student (first year through Masters) and one session per year were replaced with VR equivalents, the initial outlay on VR hardware and software development would be recouped in just over two years. Parenthetically, we estimate based on current availability of previous VR hardware and software products, that the technology for implementing VR teaching and learning is future-proofed for at least five years. Note that we have assumed that the maintenance of VR headsets is minimal, provided they are regularly cleaned and their proper use is adequately instructed.

Let us now consider how VR may add value to a course. The number of opportunities students have to enhance their practical skills are limited, in every institution, by the costs of running conventional laboratory and field sessions. Running supplementary VR practicals would therefore give students extra practical experience and hence enhance their experience. Furthermore, VR practicals allow students to process 
crime scenes in isolation, which despite being a key requirement for working in the field, is rarely offered in higher education outside of the confines of an examination, again due to monetary restrictions. It would not be infeasible to consider situations where students could be allowed to borrow headsets for use in their own time, thereby allowing engaged students to get more practice as well as enabling those who may be experiencing temporary difficulties with regular attendance (e.g. due to personal and/or health issues).

\subsection{Limitations 83 Further Development}

The development cycle for this beta test was limited by both time and funding, hence the app should be considered a minimum working example in terms of features and assets. In addition to areas for further development already raised, user comments highlight the importance of several factors for maintaining immersion and hence supporting learning. These include giving mixed control schemes and other quality-of-life improvements, accurately representing field techniques and equipment (via suitable three-dimensional models) and allowing users as much freedom of movement and interaction as possible.

Although we found the use of VR to be highly profitable, its limitations must be considered by learning providers before implementation. Full immersion in the experience will always be limited by both software and hardware, hence we do not suggest that VR should or will ever fully replace conventional practical-based learning. Furthermore, initial financial investment in VR hardware prior to implementation is significant, as is the cost of development, at least whilst VR software development remains the domain of specialised skill sets. We recommend, therefore, that VR should be considered as an adjunct to teaching and learning and applied for enhancing outcomes, especially in cases where factors such as accessibility are crucial.

\subsection{Conclusion}

We find that VR technologies are an engaging and effective method for imparting practical crime scene skills. Furthermore they are more accessible and may potentially offer a wider range of more cost-effective solutions than conventional methods. Whilst we don't suggest that VR should replace conventional practical experience, we find that it is a valuable adjunct to Forensic Science education that will enhance both student satisfaction and learning outcomes.

[1] Chartered Society of Forensic Sciences, Component standards v2017-1, 2020. URL: https://www.csofs .org/Component-Standards-Revised-2017.

[2] M. Garcia-Bonete, M. Jensen, G. Katona, A practical guide to developing virtual and augmented reality exercises for teaching structural biology, Biochemistry and Molecular Biology Education 47 (2018) 16-24. doi:10.1002/bmb.21188. 
[3] R. Mintz, S. Litvak, Y. Yair, 3d-virtual reality in science education: An implication for astronomy teaching, Journal of Computers in Mathematics and Science Teaching 20 (2001) 293-305.

[4] K. Stepan, J. Zeiger, S. Hanchuk, A. Del Signore, R. Shrivastava, S. Govindaraj, A. Iloreta, Immersive virtual reality as a teaching tool for neuroanatomy, International Forum of Allergy \& Rhinology 7 (2017) 1006-1013. doi:10.1002/alr.21986.

[5] S. Bennie, K. Ranaghan, H. Deeks, H. Goldsmith, M. O'connor, A. Mulholland, D. Glowacki, Teaching enzyme catalysis using interactive molecular dynamics in virtual reality, Journal of Chemical Education 96 (2019) 2488-2496. doi:10.1021/acs.jchemed.9b00181.

[6] A. Samosorn, G. Gilbert, E. Bauman, F. Khine, D. McGonigle, Teaching airway insertion skills to nursing faculty and students using virtual reality: A pilot study, Clinical Simulation in Nursing 39 (2020) 18 - 26. doi:https://doi.org/10.1016/j.ecns.2019.10.004.

[7] M. Mentzelopoulos, J. Parrish, P. Kathrani, D. Economou, Revrlaw: An immersive way for teaching criminal law using virtual reality, in: C. Allison, L. Morgado, J. Pirker, D. Beck, J. Richter, C. Gütl (Eds.), Immersive Learning Research Network, Springer International Publishing, Cham, 2016, pp. 73-84.

[8] W. Leung, F. Blauw, An augmented reality approach to delivering a connected digital forensics training experience, in: K. J. Kim, H.-Y. Kim (Eds.), Information Science and Applications, Springer Singapore, Singapore, 2020, pp. 353-361.

[9] U. Karabiyik, C. Mousas, D. Sirota, T. Iwai, M. Akdere, A virtual reality framework for training incident first responders and digital forensic investigators, in: G. Bebis, R. Boyle, B. Parvin, D. Koracin, D. Ushizima, S. Chai, S. Sueda, X. Lin, A. Lu, D. Thalmann, C. Wang, P. Xu (Eds.), Advances in Visual Computing, Springer International Publishing, Cham, 2019, pp. 469-480.

[10] C. Park, Engaging students in the learning process: the learning journal, Journal of Geography in Higher Education 27 (2003) 189-199.

[11] D. Lucardie, The impact of fun and enjoyment on adult's learning, Procedia - Social and Behavioral Sciences 142 (2014) 439 - 446. doi:https://doi.org/10.1016/j.sbspro.2014.07.696, the Fourth International Conference on Adult Education, Romania 2014.

[12] M. Kintu, C. Zhu, E. Kagambe, Blended learning effectiveness: the relationship between student characteristics, design features and outcomes, International Journal of Educational Technology in Higher Education 14 (2017). doi:https://doi.org/10.1186/s41239-017-0043-4. 
[13] S. Weech, S. Kenny, M. Barnett-Cowan, Presence and cybersickness in virtual reality are negatively related: A review, Frontiers in Psychology 10 (2019) 158. doi:10.3389/fpsyg.2019.00158. 